\title{
SOME ASPECTS OF THE THERMODYNAMIC LIMIT
}

\author{
A. MÜNSTER \\ Institute of Theoretical Physical Chemistry, University of Frankfurt (Main), \\ Graefstrasse 38, 6 Frankfurt (Main), Western Germany
}

\begin{abstract}
The historical development of statistical mechanics over the last hundred years is outlined, culminating in the work of Ruelle and of Fisher in 1963. The 'thermodynamic limit' is defined and conditions on intermolecular potentials and limit theorems are next examined as preliminaries to a detailed consideration of the associated equivalence problem. In conclusion the limits of applicability of thermodynamics are noted.
\end{abstract}

\section{INTRODUCTION}

In the early days of statistical mechanics, it was recognized that the laws of thermodynamics could be derived from molecular theory only for systems containing a large number of particles, i.e. for macroscopic systems. This was pointed out by Boltzmann ${ }^{1}$ when introducing Stirling's formula, and it has been stated more explicitly by Gibbs ${ }^{2}$ in the foreword to his famous treatise on Elementary Principles in Statistical Mechanics. He writes: 'The laws of thermodynamics, as empirically determined, express the approximate and probable behaviour of systems of a great number of particles, or, more precisely, they express the laws of mechanics as they appear to beings who have not the fineness of perception to enable them to appreciate quantities of the order of magnitude of those which relate to single particles, and who cannot repeat their experiments often enough to obtain any but the most probable results. The laws of statistical mechanics apply to systems of any number of degrees of freedom and are exact.... The laws of thermodynamics may be easily obtained from the principles of statistical mechanics of which they are an incomplete expression'. Gibbs ${ }^{2}$ has also shown that in defining analogues of thermodynamic quantities conceptual difficulties can be avoided only if the number of particles is assumed to be very large. This may be illustrated by means of an example. If the statistical analogue of the entropy is defined by the canonical and the grand canonical ensemble respectively, it turns out that these quantities are different from each other. It can be shown, however, that the grand canonical entropy may be expressed as the most probable value of the canonical quantity plus a term depending on the number of particles which becomes completely negligible for macroscopic systems. It is seen that Gibbs always had in mind a macroscopic but finite system. He was in 


\section{A. MÜNSTER}

fact not interested in the problem how the laws of thermodynamics could be obtained from statistical mechanics in full mathematical rigour. This, however, is precisely the question on which the more recent development has focused attention and which nowadays is usually termed as 'asymptotic problem of statistical mechanics'.

In this new field the first great success was achieved in 1922 by Darwin and Fowler ${ }^{3,4}$ who treated quantum systems of non-interacting particles. The analogous problem in classical statistics was solved by Khinchin ${ }^{5}$ whose book was published in the U.S.A. in 1949. In spite of significant differences in the details of the mathematical technique, the underlying basic idea is the same in both methods, namely the use of a generating function which is nothing else but the canonical partition function. This entails that in the final result the thermodynamic relation between the entropy and the Helmholtz free energy appears as the leading term of an asymptotic expansion. For later considerations, it will be useful to write down the essential results in a rather simple form. Let $\Phi(E)$ denote the Gibbs energy function, $Q(\beta)$ with $\beta=1 / k T$ the canonical partition function and $\bar{E}$ the áverage energy of a system of the canonical ensemble. Then in a first step one obtains for large $N$, if $N$ is the number of particles,

$$
W(E)=\frac{\exp (-\beta E) \exp (\Phi(E))}{Q(\beta)}=\frac{1}{(2 \pi B)^{\frac{1}{2}}} \exp \left(-\frac{1}{2} \frac{(E-\bar{E})^{2}}{B}\right)+\mathrm{O}\left(N^{-1}\right)
$$

where $W(E)$ is the probability density (frequency function) of the energy in the canonical ensemble. It is seen that for very large systems, this probability density tends to a Gaussian. Now let us assume that the average energy $\bar{E}$ equals the microcanonical energy $E^{\star}$. Then, on taking logarithms and dividing by $N$, we obtain from equation 1

$$
N^{-1} \Phi(\bar{E})=N^{-1} \ln Q(\beta)+N^{-1} \beta \bar{E}+\mathrm{O}\left(N^{-1} \ln N\right)
$$

or, introducing thermodynamic quantities per particle,

$$
T s(e, v)=-f(T, v)+e+\mathrm{O}\left(N^{-1} \ln N\right)
$$

Now, in the case of non-interacting particles, for $N, E \rightarrow \infty$ at $v=$ const. on the RHS the existence of the limit functions is trivial. Thus passing to the limit we may safely conclude that on the LHS the limit function exists as well which immediately leads to the well-known thermodynamic relation between entropy and Helmholtz free energy.

This was the state of affairs in 1949. It is now easy to see that the extension of the aforementioned results to interacting particles and other ensembles will meet with two additional problems. In the first place, the derivation of equation 1 is based on the central limit theorem of probability theory which means that the total energy $E$ is assumed to be a sum of $N$ independent random variables. Obviously this is no longer true in the case of interacting particles. Secondly, for interacting particles the existence of the limit functions on the RHS of equations 2 and 3 is by no means trivial but will depend rather on the details of the intermolecular interaction.

In the following period which starts in 1949 with van Hove's ${ }^{6}$ paper on a limit theorem for the canonical ensemble, several attempts were made to 
solve the aforementioned problems for special cases. Some important results have been obtained but we cannot go into the details here and the interested reader is referred to the literature ${ }^{7}$. We shall turn rather to the most recent development which was induced by the fundamental work of Ruelle $^{8}$ and Fisher ${ }^{9}$ in 1963.

\section{DEFINITIONS. STATEMENT OF THE PROBLEM}

In what follows we shall frequently denote extensive parameters by $X_{i}$ and intensive parameters by $\boldsymbol{P}_{\boldsymbol{i}}$ Remembering that in the Gibbs fundamental equation the entropy is a function of extensive state variables only, any Massieu-Planck function $\Phi_{k}$ depending on $k$ intensive parameters appears as a $k$-fold Legendre transform of the entropy, viz.

$$
\Phi_{k}=S-\sum_{i=1}^{k} P_{i} X_{i}
$$

satisfying the differential equation

$$
\mathrm{d} \Phi_{k}=-\sum_{i=1}^{k} X_{i} \mathrm{~d} P_{i}+\sum_{j=k+1}^{r} P_{j} \mathrm{~d} X_{j}
$$

where

$$
\begin{aligned}
\frac{\partial \Phi_{k}}{\partial P_{i}}=-X_{i}, \quad \frac{\partial \Phi_{k}}{\partial X_{j}} & =P_{j} \\
& (i \leqslant k<j)
\end{aligned}
$$

Turning to statistical mechanics we first observe that any statistical ensemble depending on $k$ intensive parameters generates the analogue of a Massieu-Planck function $\Phi_{k}$ by the equation

$$
\Phi_{k}=\ln \Xi_{k}
$$

where $\Xi_{k}$ is the partition function of the ensemble and, for the sake of simplicity, Boltzmann's constant has been put equal to unity. The function $\Phi_{k}$ as defined by equation 7 satisfies a differential equation of the form of equation 5 . On the other hand, in the semi-classical approximation we have

$$
\exp \left(\Phi_{k}\right)=\int_{0}^{\infty} \ldots \int_{0}^{\infty} \exp \left(-\sum_{i=1}^{k} P_{i} X_{i}\right) \exp (\Phi) \prod \mathrm{d} X_{i}
$$

That is to say that the partition function of the $k$-ensemble is the $k$-fold Laplace transform of the microcanonical partition function.

It is easy to see and it can be shown explicitly that for finite systems equations 4 and 8 are not consistent with each other. We therefore consider a sequence of systems characterized by the variable $X_{r}^{(n)}=n X_{r}^{(1)}$ where $X_{r}^{(1)}$ is some fixed reference value and $n \geqslant 1$. Furthermore we introduce the notation

$$
\varphi_{k}^{(n)}=\Phi_{k}^{(n)} / X_{r}^{(n)}, \quad x_{j}=X_{j}^{(n)} / X_{r}^{(n)}
$$




\section{A. MÜNSTER}

By 'thermodynamic limit' we mean the limit process

$$
\begin{array}{ll}
n \rightarrow \infty \quad \text { for } \quad P_{i}=\text { const., } & (i=1,2, \ldots, k) \\
& x_{j}=\text { const., } \quad(j=k+1, \ldots n-1)
\end{array}
$$

Thus what has been called above the 'asymptotic problem' amounts to a study of the thermodynamic limit comprising the following subproblems:

(a) Existence of the limit $\lim _{n \rightarrow \infty} \varphi_{k}^{(n)}$ and its derivatives (limit theorems)

(b) Consistency of the thermodynamic quantities defined with the aid of various ensembles (equivalence problem)

(c) Thermodynamic stability

(d) Phase transitions.

These questions are closely connected with one another and therefore cannot be discussed independently. Here, however, we are mainly concerned with the equivalence problem. Questions (a) and (c) will be touched upon rather briefly whereas we shall leave out the problem of phase transitions.

\section{CONDITIONS ON INTERMOLECULAR POTENTIALS. LIMIT THEOREMS}

The existence of the limit functions will depend on assumptions on the intermolecular potentials. For one thing, the forces of attraction could be so strong that the system 'collapses' as the number of particles increases, so that $\varphi_{k}^{\infty}$ diverges to $+\infty$. On the other hand, the forces of repulsion could decrease so little with increasing separation that $\varphi_{k}^{\infty}$ diverges to $-\infty$. Following Fisher $^{9}$, we therefore make the following assumptions:

\section{(A) Condition of stability}

For the potential energy of a system of $N$ interacting particles $U^{(N)}$ there exists a lower bound

$$
U^{(N)} \geqslant-N u_{A}
$$

for all values of the coordinates and all $N$, where $u_{A}$ is a fixed natural number. Potentials which satisfy condition A are called stable potentials.

\section{(B1) Condition of weak tempering}

Let us imagine that the $N$ particles have been split up into two groups of $N_{1}$ and $N_{2}$ particles with coordinates $\vec{q}_{i}$ and $\vec{q}_{j}^{\prime}$ respectively. The interaction energy between these groups will be denoted by $U^{\left(N_{1} \cdot N_{2}\right)}\left(\vec{q}_{i} ; \vec{q}_{j}^{\prime}\right)$. Then for all $N_{1}$ and $N_{2}$ and some arbitrary fixed $R_{0}$ and $u_{B}$ and $\varepsilon>0$

$$
U^{\left(N_{1} . N_{2}\right)}\left(\vec{q}_{i} ; \vec{q}_{j}^{\prime}\right) \leqslant \frac{N_{1} N_{2} u_{B}}{R^{3+\varepsilon}}
$$

if $\left|\vec{q}_{i}-\vec{q}_{j}^{\prime}\right| \geqslant R \geqslant R_{0}$ holds for all $i$ and $j$ and $\left(N_{1}+N_{2}\right) / R^{3+\varepsilon}$ is sufficiently small. 


\section{(B2) Condition of strong tempering}

Under the same assumptions as before

$$
U^{\left(N_{1} . N_{2}\right)}\left(\vec{q}_{i}, \vec{q}_{j}\right) \leqslant 0
$$

whenever $\left|\vec{q}_{i}-\vec{q}_{j}^{\prime}\right| \geqslant R_{0}$ for all $i$ and $j$.

On the basis of the assumptions $\mathrm{A}$ and $\mathrm{Bl}, \mathrm{Fisher}^{9}$ has proved that for the canonical ensemble the limit $\varphi_{k}^{\infty}$ exists and is a continuous convex and nondecreasing function of the volume per particle $v$. From the convexity property which is essentially equivalent to thermodynamic stability conditions, existence and properties of the derivatives are obtained by the use of wellknown theorems on convex functions ${ }^{10}$. It cannot be shown, however, that we have

$$
\frac{\partial \varphi_{k}^{\infty}}{\partial v}=\lim _{n \rightarrow \infty} \frac{\partial \varphi_{k}^{(n)}}{\partial v}
$$

as required for a complete statistical foundation of thermodynamics. Mathematically this shortcoming arises from the fact that convexity can only be proved for the limit function $\varphi_{k}^{\infty}$.

Analogous results have been obtained for the grand canonical ensemble ${ }^{9}$ whereas van der Linden ${ }^{11}$ has proved a limit theorem for the microcanonical ensemble on the basis of assumptions A and B2.

\section{EQUIVALENCE PROBLEM}

The formal nature of the equivalence problem becomes immediately obvious from the comparison of equations 4 and 8 . We have to prove that, at the thermodynamic limit, the Laplace transformation of the statistical partition functions reduces asymptotically to the Legendre transformation of the Massieu-Planck functions. We may still ask, however, for the physical meaning of this problem. There are two possible answers which of course only elucidate two aspects of the same situation. In the first place we may consider that any statistical ensemble is linked conceptually with a particular physical situation of the system of interest. The microcanonical ensemble represents an isolated system, the canonical ensemble a system in contact with a heat bath, and similarly for the other ensembles. The laws of thermodynamics, however, do not depend on a particular physical situation. Thus it must be shown that these 'boundary conditions' become meaningless at the thermodynamic limit. On the other hand we may look on the asymptotic expansion 3. It can be shown that the higher order terms arise from statistical fluctuations. In thermodynamics, however, this concept does not appear at all. We therefore must prove that fluctuations vanish asymptotically at the thermodynamic limit.

The aforementioned physical aspects correspond closely to the mathematical methods which in recent years have been applied to the general treatment of the equivalence problem. In the following we shall describe the essential features of these methods from a more physical point of view, again leaving out the mathematical details. 


\section{(i) Method of van der Linden and Mazur}

The first method due to van der Linden and Mazur ${ }^{11.12}$ is based first on assumptions A and B2 of Section III and secondly on certain inequalities for the microcanonical ensemble. These essentially state that the phase volume is a never negative and never decreasing convex function of the energy. In a first step it is shown that the limit theorem for the microcanonical ensemble mentioned in Section III generates an analogous limit theorem for the canonical ensemble. We briefly sketch the main idea of the proof. Let us imagine that the system is divided into two subvolumes with numbers of particles and energies $N_{1}, E_{1}$ and $N-N_{1}, E-E_{1}$ respectively. Then using a well-known formula for the microcanonical ensemble and the aforementioned inequalities it is easily established that we must have for the phase volume

$$
\Omega^{\star}(E) \geqslant \int_{0}^{E} \exp \left(\Phi_{1}\left(E_{1}\right)\right) \Omega_{2}^{\star}\left(E-E_{1}\right) \mathrm{d} E_{1}
$$

This property leads in fact to the limit theorem for the microcanonical ensemble. Since the right hand member is a convolution integral, we obtain with the aid of the convolution theorem for the Helmholtz free energy per particle

$$
-N \mathrm{f}(N) \leqslant-N_{1} \mathrm{f}\left(N_{1}\right)-\left(N-N_{1}\right) \mathrm{f}\left(N-N_{1}\right)
$$

Functions satisfying an inequality of this form are called subadditive functions. For these we have a limit theorem ${ }^{13}$ which combined with assumption A leads to the desired limit theorem for the canonical ensemble.

In dealing with the equivalence problem itself the following ingenious device is used. First we generalize the above division to a division into $n$ subsystems which are assumed to have equal energies $E^{\star}$, equal numbers of particles $N^{\star}$ and equal volumes $V^{\star}$. Then, according to the theory of the microcanonical ensemble and condition B2 it must be true that

$$
s_{n}^{\star}\left(E^{\star}, N^{\star}, V^{\star}\right) \geqslant{ }^{(n)} s^{\star}\left(E^{\star}, N^{\star}, V^{\star}\right) \geqslant s^{\star}\left(E^{\star}, N^{\star}, V^{\star}\right)
$$

The left hand member of this inequality is simply the entropy of the original system divided by the number of subsystems. In the second member the interaction between the subsystems is neglected but not the energy distribution between them, i.e. they are assumed to be in thermal equilibrium. In the last member the subsystems are considered to be isolated. Thus we have three different physical situations and we shall show that these differences become meaningless at the thermodynamic limit.

The second member of expression 17 refers to an 'ideal system'. Hence, considering the subsystems as 'particles', we may apply Khinchin's argument. Passing to the limit $n \rightarrow \infty$ for fixed $N^{\star}$ [which does not affect the last member of 17] we then obtain from equation 3 and the limit theorem for the microcanonical ensemble

$$
s^{\infty}(e, v) \geqslant-\beta \mathrm{f}(\beta, v, N)+\beta \bar{e}(\beta, v, N) \geqslant s(e, v, N)
$$

where $N^{\star}$ has been replaced by $N$ and quantities per particle have been introduced. Next we study the thermodynamic limit of a subsystem $(N \rightarrow \infty$, $\beta=$ const., $v=$ const.). Under this process the first member becomes simply $s^{\infty}[e(\beta, v), v]$ and the last member converges to the same limit. 
Hence, making use of the limit theorem for the canonical ensemble, we obtain

$$
s^{\infty}[e(\beta, v), v]=-\beta \mathrm{f}^{\infty}(\beta, v)+\beta e^{\infty}(\beta, v)
$$

which is a special case of the thermodynamic relation 4 . This argument can be carried through for all conceivable ensembles although the details become more complicated. Under stronger assumptions about the intermolecular forces (two-body central forces satisfying an additional condition) van der Linden ${ }^{14}$ has also proved equation 14.

\section{(ii) Method of Still, Haubold and Münster}

The other approach due to Still, Haubold and Münster ${ }^{15}$ is essentially a generalization of Khinchin's method to interacting particles and any conceivable ensemble. In comparison with the first method, assumptions about intermolecular forces here enter only via limit theorems but they are not used explicitly. Furthermore thermodynamic relations are obtained as leading terms of asymptotic expansions. The underlying assumptions are essentially the existence of a limit theorem for the function $\varphi_{l+1}(P)$ and the exclusion of phase transition points.

As explained in section $I$, we have first to prove that the frequency function $W(y)$ with

$$
y=(X-\bar{X}) / B^{\frac{1}{2}}
$$

tends to a Gaussian at the thermodynamic limit. This problem is of considerable interest in itself since in many applications the frequency function is assumed to be a Gaussian. Now it can be shown without difficulty that the sequence of characteristic functions

$$
\psi^{(n)}(t)=\int_{-\infty}^{+\infty} \mathrm{e}^{i t y} W^{(n)}(y) \mathrm{d} y
$$

for $n \rightarrow \infty$ converges to a Gaussian in any finite $t$-interval and, moreover, that the moments of the frequency function converge to the moments of a Gaussian with dispersion one. From this result, however, we cannot conclude that the functions

$$
W^{(n)}(y)=\frac{1}{2 \pi} \int_{-\infty}^{+\infty} \mathrm{e}^{-i t y} \psi^{(n)}(t) \mathrm{d} t
$$

converge to a Gaussian. The reason is that convergence of the $\psi^{(n)}(t)$ has been proved only for finite $t$-intervals. Thus in performing the integral of equation 22 we must have some knowledge about the 'tail' of the integrand. As shown by Mazur and van der Linden ${ }^{16}$ this knowledge is indeed available for the canonical ensemble but unfortunately this is not true in the general case. To overcome this difficulty we replace the original frequency function by a 'smoothed' frequency function $W_{s}^{(n)}(y)$ which is obtained from the former by convolution with an appropriate smoothing function $S^{(n)}(y)$ in such a way that the smoothed frequency function remains normalized and non-negative. Thus we define

$$
W_{s}^{(n)}(y)=\int_{-\infty}^{+\infty} W^{(n)}\left(y^{\prime}\right) S^{(n)}\left(y-y^{\prime}\right) \mathrm{d} y^{\prime}
$$

It is easy to see that the smoothing procedure is nothing else but a local 


\section{A. MÜNSTER}

averaging. This, however, means that the characteristic function is damped for large values of $t$. Since we know $S^{(n)}(y)$ explicitly this gives us the required knowledge about the tail. Therefore we are now able to show that for large $n$ we have

$$
W_{s}^{(n)}(X)=\frac{1}{(2 \pi B)^{\frac{1}{2}}} \exp \left(-\frac{1(X-\bar{X})^{2}}{2}\right)\left[1+\mathrm{O}\left(n^{-1} \ln ^{2} n\right)\right]
$$

which is the generalization of equation 1 .

Although 'smoothing' or 'coarse graining' is nothing new in statistical mechanics we still wish to give some justification for the above procedure. This is achieved by showing rigorously that the functions $W^{(n)}(y)$ and $W_{s}^{(n)}(y)$, at the thermodynamic limit, cannot be distinguished by any conceivable macroscopic measurement. From equation 24 we obtain by a straightforward argument

$$
\varphi_{l}^{n}=\varphi_{l+1}^{n}+P_{l+1} \bar{x}_{l+1}+\mathbf{O}\left(n^{-1} \ln ^{2} n\right)
$$

which on passing to the limit $n \rightarrow \infty$ yields again the thermodynamic relation 4 and at the same time proves the existence of $\varphi_{l}^{\infty}$. Formally equation 25 was proved only for smoothed functions. But it can be shown rigorously that $\varphi_{l+1, s}^{(n)}=\varphi_{l+1}^{(n)}$ for all $n$. Then it follows from the uniqueness of the Legendre transformation, that there is one and only one function $\varphi_{l}^{\infty}$ satisfying the thermodynamic equation 4 . Hence we are left with two possibilities. Either the 'true' limit function $\varphi_{l}^{\infty}$ does not exist or does not satisfy equation 4. Then 'smoothing' is a necessary step in the foundation of thermodynamics. Or the 'true' limit function $\varphi_{l}^{\infty}$ satisfies equation 4 as well, then it cannot be distinguished from the smoothed function. Indeed if we assume existence of a limit theorem for $\varphi_{l}$ (which has been avoided so far) then we can prove that $\varphi_{l}^{\infty}=\varphi_{l s}^{\infty}$ almost everywhere.

Similar results are obtained for the derivatives of $\varphi_{l}^{\infty}$. In particular it can be shown that differentiation and passage to the thermodynamic limit commute. From this thermodynamic stability conditions are obtained in the general form first given by Schottky, Ulich and Wagner ${ }^{17}$ and it is shown that they are identical with conditions for the statistical fluctuations.

\section{LIMITS OF APPLICABILITY OF THERMODYNAMICS}

Up to now we have exclusively treated the formal derivation of thermodynamics from statistical mechanics which necessarily requires the introduction of the concept of an infinitely large system. However, experimental physics always deals with finite systems, so that we can expect this to impose certain bounds on the applicability of the thermodynamic formalism. These are determined by the neglected terms in equations 3 and 25 which have their origin in statistical fluctuations. Thus the domain of validity of thermodynamics is determined by the condition that fluctuations are negligible with respect to the accuracy of measurements. In most cases this will be true for macroscopic systems. Planck ${ }^{18}$, however, has already discussed an interesting case where thermodynamics breaks down even for finite macroscopic systems. 
Consider a Debye crystal, say a cube of edge length $1 \mathrm{~cm}\left(N \approx 10^{21}\right)$ with characteristic temperature $\Theta_{D}=10^{2}{ }^{\circ} \mathrm{K}$ which has been cooled to a temperature of $10^{-5} \mathrm{~K}$. Then from a general formula derived by Münster ${ }^{19}$ we have

$$
\frac{1}{T}=\left(\frac{\partial S}{\partial E}\right)_{0}+\frac{1}{2} \bar{E}_{D}^{2}\left(\frac{\partial^{3} S}{\partial E^{3}}\right)_{0} \frac{\left(\overline{E-\bar{E}_{D}}\right)^{2}}{-\bar{E}_{D}^{2}}
$$

with

$$
\bar{E}_{D}=\frac{3 \pi^{4}}{5} N k \frac{T^{4}}{\Theta_{D}^{3}}, \bar{E}=\bar{E}_{D}+\frac{9}{8} N k \Theta_{D}
$$

Numerical calculation shows that both terms of the RHS of equation 26 are of the same order of magnitude $10^{5}\left[{ }^{\circ} \mathrm{K}\right]^{-1}$. Thus, as stated already by Planck ${ }^{18}$, near the absolute zero the concepts of entropy and absolute temperature are no longer uniquely definable because fluctuations of energy become important. It is obvious that this has some bearing on the interpretation of Nernst's heat theorem ${ }^{20}$.

\section{REFERENCES}

1 L. Boltzmann, S.B. Akad. Wiss. Wien, 76, 373 (1877) [Wissensch. Abh., Vol. II, p 177. New York (1968)].

2 J. W. Gibbs, Elementary Principles in Statistical Mechanics. New Haven (1902) [Collected works, Vol. II, p VIII, 203, New Haven (1948)].

3 C. S. Darwin and R. H. Fowler, Phil. Mag. 44, 450 and 823 (1922); 45, 1 (1923).

4 R. H. Fowler, Statistical Mechanics, 2nd ed. Cambridge University Press: London (1936).

5 A. J. Khinchin, Mathematical Foundation of Statistical Mechanics. New York (1949).

${ }^{6}$ L. van Hove, Physica, 15, 951 (1949).

7 A. Münster, Statistical Thermodynamics, Vol. I. Springer: Berlin and New York (1969).

8 D. Ruelle, Helv. Phys. Acta, 36, 183 (1963).

9 M. E. Fisher, Arch. Ration. Mech. Anal. 17, 377 (1964).

10 G. H. Hardy, J. E. Littlewood and G. Pólya, Inequalities, 2nd ed., Cambridge University Press: London (1952).

11 J. van der Linden, Physica, 32, 642 (1966).

12 J. van der Linden and P. Mazur, Physica, 36, 491 (1967).

13 E. Hille and R. S. Phillips, Functional Analysis and Semi-groups. Providence (1957).

${ }_{14}$ J. van der Linden, Physica, 38, 173 (1968).

15 E. Still, K. Haubold and A. Münster, Z. Naturforschung, 24a, 201 and 412 (1969).

16 P. Mazur and J. van der Linden, J. Math. Phys. 4, 271 (1963).

17 W. Schottky, H. Ulich and C. Wagner, Thermodynamik. Springer: Berlin (1929).

18 M. Planck, Theorie der Wärmestrahlung, 4te Aufl., p 218. Springer: Berlin (1921).

19 A. Münster, Z. Phys. 136, 179 (1953).

${ }^{20}$ M. J. Klein, The Laws of Thermodynamics. (R.C. Scu. Intern. Fis. 'Enrico Fermi', Corso X) Bologna (1960). 